\title{
Impact of bariatric surgery on the management of type 2 diabetes mellitus in Singapore
}

\author{
Phong Ching Lee ${ }^{1,3}$, MBChB, MRCP, Kwang Wei $\underline{T h a m}^{1,3}$, MB BCh BAO, Hong Chang $\underline{\text { Tan }},{ }^{1,3}$, MBBS, MRCP,
} Shanker Pasupathy ${ }^{2,3}$, MBBS, FRCS

INTRODUCTION Obesity is a risk factor for type 2 diabetes mellitus (T2DM). Metabolic-bariatric surgery (MBS) results in significant weight loss with dramatic improvement in T2DM. This study analysed the effects of MBS on patients with T2DM in a tertiary centre in Singapore.

METHODS Individuals with T2DM who underwent MBS in a single centre from September 2008 to May 2012 , with at least 12 months of regular follow-up, were included in our study. The primary outcome measure was good glycaemic control (glycated haemoglobin $[\mathrm{HbA1c}]<6.5 \%$, with or without medications) 12 months after surgery. Secondary outcome measures were partial DM remission (fasting blood glucose [FBG] $<7.0 \mathrm{mmol} / \mathrm{L}$ and $\mathrm{HbA} 1 \mathrm{C}<6.5 \%$ without DM medications), complete DM remission (FBG $<5.6 \mathrm{mmol} / \mathrm{L}$ and $\mathrm{HbA} 1 \mathrm{c}<6.0 \%$ without DM medications), weight, body mass index, blood pressure, and fasting serum lipid, serum glucose and serum insulin levels.

RESULTS Of the 19 patients who met the inclusion criteria, 14 underwent gastric bypass and 5 underwent sleeve gastrectomy. At 12 months postoperatively, 17 (89.5\%) patients achieved good glycaemic control. DM remission was achieved in $14(73.7 \%)$ patients, with $10(52.6 \%)$ attaining complete remission.

CONCLUSION In Singapore, MBS is an effective treatment modality for obese patients with T2DM. Despite the small sample size and lack of matched controls, the present study suggests that MBS is effective in achieving significant weight loss and eliciting a significant and sustainable improvement in the glycaemic control of patients with T2DM, for up to 12 months.

Keywords: bariatric surgery, diabetes mellitus, metabolic, obesity, remission

\section{INTRODUCTION}

The association between obesity and type 2 diabetes mellitus (T2DM) is well established, with obesity being a major modifiable risk factor in T2DM development. ${ }^{(1)}$ The main goal of achieving good glycaemic control is to reduce the risk of DM-related micro- and macrovascular complications, which contribute to the major morbidity and mortality in patients with T2DM. ${ }^{(2,3)}$ Despite an increasing armamentarium of advanced therapies for the treatment of T2DM, the number of patients achieving good glycaemic control remains dismally low. ${ }^{(4)}$

In 1995, Pories et al observed that gastric bypass (GB) surgery for morbid obesity could achieve and sustain euglycaemia in $83 \%$ of patients with T2DM and $99 \%$ of patients with glucose impairment, even 14 years after surgery. ${ }^{(5)}$ Since then, there has been a growing body of evidence that bariatric surgery, originally intended to effect sustainable and drastic weight loss in the morbidly obese, has substantial beneficial effects on obesity-related metabolic conditions, improving glycaemic control in $\mathrm{T}^{2} \mathrm{DM}^{(6)}$ and reducing rates of cardiovascular disease ${ }^{(7)}$ and death. ${ }^{(8)}$ As a result, bariatric surgery is now known as metabolic-bariatric surgery $(\mathrm{MBS})^{(9)}$ and increasingly considered in the management of obesity-related metabolic disease, particularly T2DM.
The robust evidence of the impact of MBS on T2DM amelioration has led major scientific organisations, such as the American Diabetes Association and the International Diabetes Federation, to recommend bariatric surgery as part of the treatment algorithm for obese patients with T2DM. ${ }^{(9)}$

In Singapore and other Asian countries, the use of MBS to combat the rising trend of obesity and T2DM has grown in popularity. From 2004 to 2009, the absolute number of bariatric surgery procedures conducted in Asia increased by 5.5 times, from 381 to 2,091 surgical procedures. ${ }^{(10)}$ Within the Asian population, bariatric surgery has also resulted in significant and sustained weight loss, with a T2DM remission rate of up to $80 \%$ in morbidly obese Asian patients. ${ }^{(11)}$

However, multi-ethnicity in Singapore poses an interesting challenge in the management of T2DM and obesity. In 2010, the National Health Survey (NHS) revealed that with the rise in obesity prevalence from $6.9 \%$ in 2004 to $10.8 \%$ in 2010, the prevalence of T2DM had risen significantly from $8.2 \%$ to $11.3 \%$, a relative rise of $38 \%$ in a timespan of just six years. ${ }^{(12,13)}$ The prevalence of T2DM was noted to be highest in Indians (17.2\%), followed by Malays (16.6\%) and Chinese $(9.7 \%) .{ }^{13)}$ The ethnic group that had the highest proportion of DM patients with poor glycaemic control was the Malays $(47.6 \%)$, followed by the Indians $(37.9 \%)$

${ }^{1}$ Department of Endocrinology, ${ }^{2}$ Department of General Surgery, ${ }^{3}$ Obesity and Metabolic Unit, Lifestyle Improvement and Fitness Enhancement Centre, Singapore General Hospital, Singapore

Correspondence: Dr Kwang Wei Tham, Senior Consultant, Department of Endocrinology, Singapore General Hospital, The Academia, 20 College Road, Singapore 169856. tham.kwang.wei@sgh.com.sg 
and Chinese (24.9\%). ${ }^{(13)}$ Hence, when exploring the various factors that impact T2DM disease control, as well as the effects of the various possible treatments, studies that are specific to the Singapore population are greatly needed.

In Singapore, a single centre's experience of seven patients with T2DM who underwent MBS found that about $29 \%$ of the patients achieved "remission" of DM, while all patients saw an improvement in their glycaemic control at 12 weeks after surgery.(14) The study, however, was small and examined only short-term data. In the present study, we aimed to evaluate the longer-term effects of MBS in patients with T2DM by analysing the 12-month data on glycaemic control and other metabolic parameters.

\section{METHODS}

This is a single-centre, prospective, observational study conducted in a tertiary hospital in Singapore. Individuals with T2DM who underwent MBS in Singapore General Hospital (SGH) from September 2008 to May 2012 were included in our study. DM was diagnosed according to the World Health Organization's proposed criteria, and patients with DM were managed by dedicated endocrinologists. Patients had to meet the criteria for MBS, which was based on Singapore's Ministry of Health guidelines, ${ }^{(15)}$ and have their medical management optimised before undergoing either laparoscopic sleeve gastrectomy (SG) or laparoscopic GB. The laparoscopic procedures were performed by a single surgeon.

Laparoscopic SG was performed as reported previously. ${ }^{(16)}$ Roux-en-Y GB was also performed laparoscopically, creating a 30-mL gastric pouch using an endoscopic stapler and biliary and alimentary limbs measuring $100 \mathrm{~cm}$ each. ${ }^{(17)}$ Patients were selected to undergo either laparoscopic SG or laparoscopic GB based on their baseline profile, and after the surgeon and endocrinologist involved had discussed the risks and benefits of each procedure with the patient. Patients who attended regular follow-up and had at least a year of follow-up data were included in the study.

The primary outcome measure was good glycaemic control at 12 months after surgery. This was defined as $\mathrm{HbA} 1 \mathrm{c}<6.5 \%$, with or without medications. The rates of DM remission were studied as a secondary measure. Using recently published consensus definitions, ${ }^{(18)}$ we defined partial DM remission as $\mathrm{HbA} 1 \mathrm{c}<6.5 \%$ and $\mathrm{FBG}$ $<7.0 \mathrm{mmol} / \mathrm{L}$, and complete remission as $\mathrm{HbA} 1 \mathrm{c}<6 \%$ and $\mathrm{FBG}<5.6 \mathrm{mmol} / \mathrm{L}$. For patients to be considered to be in remission, they must also not be on any DM medications. Other secondary outcome measures included weight, body mass index (BMI), blood pressure, fasting serum lipid (total cholesterol, triglycerides, high-density lipoprotein [HDL] and low-density lipoprotein) levels, as well as fasting serum glucose and insulin levels. To estimate insulin resistance, homeostatic model assessment
Table I. Baseline characteristics of patients $(n=19)$.

\begin{tabular}{lc}
\hline Characteristic & No. (\%) \\
\hline Age* (yrs) & $44(31-63)$ \\
Female gender & $11(58)$ \\
Race & \\
Chinese & $9(47)$ \\
Malay & $5(26)$ \\
Indian & $4(21)$ \\
Eurasian & $1(5)$ \\
Type of procedure & \\
Gastric bypass & $14(74)$ \\
Sleeve gastrectomy & $5(26)$ \\
Length of stay* (days) & $4(3-14)$ \\
Body mass index* (kg/m $\left.{ }^{2}\right)$ & $39.7(29.2-69.0)$ \\
Comorbidities & \\
Hypertension & $16(84)$ \\
Hyperlipidaemia & $14(74)$ \\
HbA1c ${ }^{\dagger}$ (\%) & $8.1 \pm 1.2$ \\
\hline
\end{tabular}

${ }^{*}$ Data is presented as median (range). ${ }^{+}$Data is presented as mean \pm standard deviation.

of insulin resistance (HOMA-IR) was calculated using the following formula: fasting insulin $\times$ fasting glucose/22.5. Measurements were taken prior to surgery and at 12 months postoperatively. Changes in the number of medications for T2DM, hypertension and dyslipidaemia before and after surgery were tracked and compared. All patients were cared for by a multidisciplinary team consisting of a surgeon, an endocrinologist, a dietitian, a physiotherapist and a psychologist throughout the entire course of the study.

All statistical analyses were calculated using the Statistical Package for the Social Sciences version 17.0 (SPSS Inc, Chicago, IL, USA). Continuous variables with a normal distribution were reported as mean \pm standard deviation. Variables with non-normal distributions were reported as a median. Categorical variables are summarised using frequencies. Paired t-test was used to compare continuous variables before surgery and 12 months after surgery. A two-sided p-value was used and $p$-values $<0.05$ were considered statistically significant.

\section{RESULTS}

A total of 127 patients underwent MBS in SGH from September 2008 to May 2012. Of these, 50 (39.4\%) had T2DM. 19 out of these 50 patients had at least 12 months of follow-up after surgery, and were thus included in our study. A majority of the 19 patients were female $(n=11)$ and the ethnicities of the patients were Chinese $(n=9)$, Malay $(n=5)$, Indian $(n=4)$ and Eurasian $(n=1)$. Of the 19 patients, 14 (74\%) underwent laparoscopic GB (GB group), while 5 (26\%) underwent laparoscopic SG (SG group). Median BMI was $39.7 \mathrm{~kg} / \mathrm{m}^{2}$ (range 29.2-69.0 kg/m²), and the majority of the patients had comorbidities such as hypertension (84\%) and hyperlipidaemia (74\%). Mean HbA1c at baseline was $8.1 \%$ (range $6.2 \%-10.1 \%$ ). 


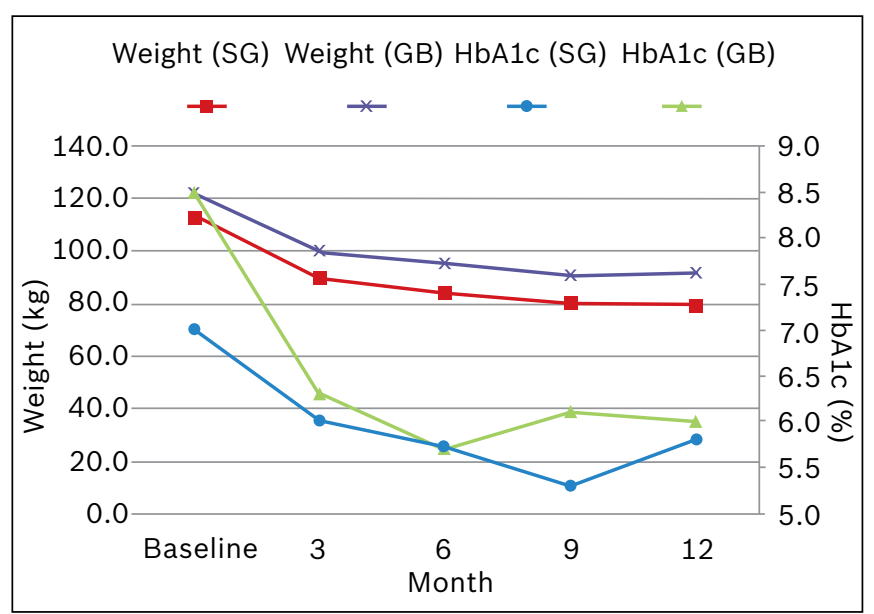

Fig. 1 Graph shows changes in the weight and HbA1c of patients after gastric bypass (GB) or sleeve gastrectomy (SG).

17 of the 19 patients had $\mathrm{HbA1c}>6.5 \%$, with the majority (11 of 19 patients) having poor glycaemic control $(\mathrm{HbA} 1 \mathrm{c} \geq 8 \%)$. The baseline characteristics of the 19 patients are summarised in Table I.

Post MBS, there was a significant decrease in the BMI of the patients, from a median of $39.7 \mathrm{~kg} / \mathrm{m}^{2}$ to $32.2 \mathrm{~kg} / \mathrm{m}^{2}$, with an average absolute weight loss (AWL) of $30.8 \mathrm{~kg}$ (25.7\% from baseline). At 12 months postoperatively, all patients who initially had a mean $\mathrm{HbA} 1 \mathrm{c}$ baseline of $8.1 \%$ had improved glycaemic control, with a mean $\mathrm{HbA1c}$ of $5.9 \%$ ( $p<0.001) .17(89.5 \%)$ of the 19 patients achieved good glycaemic control, with $\mathrm{HbA} 1 \mathrm{c}$ in the normal range $(<6.5 \%)$, which was sustained at 12 months. Of these 17 patients, $14(82.3 \%)$ were not on any DM medications, while $3(17.6 \%)$ were on metformin only. Nine $(47.3 \%)$ of the 19 patients who were on insulin (average daily dose of 86 units) prior to MBS still had poor glycaemic control, with a mean $\mathrm{HbA} 1 \mathrm{c}$ of $8.6 \%$ (range $7.2 \%-10.1 \%$ ). These nine patients were able to achieve a mean HbA1c of $6.3 \%$ without insulin treatment at 12 months postoperatively.

Fig. 1 illustrates the changes in weight and $\mathrm{HbA} 1 \mathrm{c}$ of the GB and SG groups at 3-month intervals from baseline. At 12 months, both groups of patients lost similar amounts of weight, with the SG group trending toward a slightly greater weight loss (AWL 28\%; $p=0.03$ ) than the GB group (AWL 22\%; p < 0.001); there was no statistical difference between the AWLs of the two groups. The GB group started out with a higher $\mathrm{HbA1c}(8.5 \%)$ than the SG group $(7.0 \%)$. At three months, there was a marked decrease in $\mathrm{HbA} 1 \mathrm{c}$ of $2.2 \%$ in the GB group vs. $1 \%$ in the SG group (Fig. 1). At 12 months, the reduction in $\mathrm{HbA} 1 \mathrm{c}$ from $8.5 \%$ to $6.0 \%$ was statistically significant in the GB group ( $p<0.001$ ), while that in the SG group was not (from $7.0 \%$ to $5.8 \% ; p=0.07)$. Of the 19 patients, $14(73.7 \%)$ achieved DM remission, of which 10 (52.6\%) achieved complete DM remission.

In the GB group, there was also a significant increase in $\mathrm{HDL}$ level from $0.98 \mathrm{mmol} / \mathrm{L}$ to $1.27 \mathrm{mmol} / \mathrm{L}(\mathrm{p}=0.004)$
Table II. Parameters at baseline and 12 months after metabolic-bariatric surgery.

\begin{tabular}{|c|c|c|c|}
\hline \multirow[t]{2}{*}{ Parameters } & \multicolumn{3}{|c|}{ Mean } \\
\hline & $\begin{array}{c}\text { All } \\
(n=19)\end{array}$ & $\begin{array}{c}\text { GB } \\
(n=14)\end{array}$ & $\begin{array}{c}\text { SG } \\
(n=5)\end{array}$ \\
\hline \multicolumn{4}{|l|}{ Body weight (kg) } \\
\hline Baseline & 120.0 & 122.4 & 111.7 \\
\hline At 12 mths & 89.2 & 92.1 & 79.0 \\
\hline Change from baseline & -30.8 & -30.3 & -32.7 \\
\hline$p$-value & $<0.001$ & $<0.001$ & 0.031 \\
\hline \multicolumn{4}{|l|}{ HbA1c (\%) } \\
\hline Baseline & 8.1 & 8.5 & 7.0 \\
\hline At 12 mths & 5.9 & 6.0 & 5.8 \\
\hline Change from baseline & -2.2 & -2.5 & -1.2 \\
\hline$p$-value & $<0.001$ & $<0.001$ & 0.073 \\
\hline \multicolumn{4}{|l|}{ FSG (mmol/L) } \\
\hline Baseline & 7.9 & 8.1 & 6.8 \\
\hline At 12 mths & 5.9 & 6.0 & 5.7 \\
\hline Change from baseline (\%) & -25 & -26 & -16 \\
\hline $\mathrm{p}$-value & 0.062 & 0.09 & 0.365 \\
\hline \multicolumn{4}{|l|}{ Triglyceride (mmol/L) } \\
\hline Baseline & 1.77 & 1.89 & 1.30 \\
\hline At 12 mths & 1.13 & 1.12 & 1.18 \\
\hline Change from baseline (\%) & -36 & -41 & -10 \\
\hline $\mathrm{p}$-value & 0.002 & 0.002 & 0.472 \\
\hline \multicolumn{4}{|l|}{ HDL-C (mmol/L) } \\
\hline Baseline & 0.97 & 0.98 & 0.95 \\
\hline At 12 mths & 1.22 & 1.27 & 1.03 \\
\hline Change from baseline (\%) & 26 & 30 & 8 \\
\hline $\mathrm{p}$-value & 0.003 & 0.004 & 0.543 \\
\hline
\end{tabular}

FSG: fasting serum glucose; HbA1c: glycated haemoglobin; HDL-C: high-density lipoprotein cholesterol

and a decrease in triglyceride levels from $1.89 \mathrm{mmol} / \mathrm{L}$ to $1.12 \mathrm{mmol} / \mathrm{L}(\mathrm{p}=0.002)$. At 12 months, there was a $78 \%$ decrease in HOMA-IR from 8.03 (at baseline) to 1.74, and the median 2-hour plasma glucose after $75 \mathrm{~g}$ oral glucose tolerance test was within the normal range of $6.1 \mathrm{mmol} / \mathrm{L}$ (interquartile range 3.6-8.7 mmol/L). Results of the other parameters studied are shown in Table II. Table III shows the medication use of the patients at baseline and at 12 months. On an average, patients were taking two glucose-lowering agents, two antihypertensives and one lipid-lowering drug (median values) prior to MBS. At 12 months, there was a significant reduction in the majority of medication classes used for glycaemic, blood pressure and lipid control (Fig. 2).

\section{DISCUSSION}

In our study, patients with T2DM showed dramatic improvements in their weight and glycaemic control after MBS. The significance of this finding carries even more weight when we take into account the marked reduction in the number of DM medications prescribed 12 months after MBS, as compared to before MBS. Weight gain, which occurs with time, is inevitable with various treatments for glycaemic control, especially if treatment is intensified to achieve tight control. This is supported by the Action to Control Cardiovascular Risk in Diabetes (ACCORD) study, in which patients with T2DM were randomised to either 


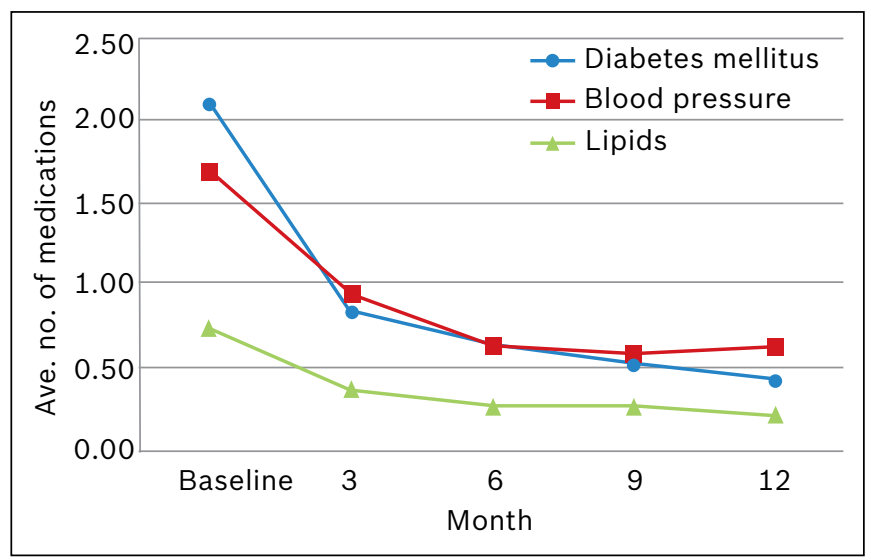

Fig. 2 Graph shows the changes in the number of medications for diabetes mellitus, blood pressure and lipid medications.

standard therapy or intensive treatment to achieve tight glycaemic control. ${ }^{(19)}$ At one year, patients who received intensive treatment achieved a median HbA1c of $6.4 \%$, while those who received standard therapy achieved a median $\mathrm{HbA} 1 \mathrm{c}$ of $7.5 \%$. A greater number of patients in the intensive treatment group $(10.3 \%$ vs. $1.2 \%)$ were on 4-5 classes of medications, including insulin. Futhermore, a greater number of patients in the intensive treatment group had significant hypoglycaemia and weight gain (> $10 \mathrm{~kg}$ ) when compared to patients who received standard therapy $(27.8 \%$ vs. $14.2 \%){ }^{(19)}$

The impact of MBS on amelioration and improvement of T2DM in our series is consistent with observational studies on bariatric procedures, which showed a T2DM remission rate of $55 \%-95 \% .{ }^{(20)}$ In our study, marked reductions in postoperative weight and $\mathrm{HbA1c}$ are observed even at three months (Fig. 1). It is well known that weight corresponds closely with glycaemic control; the Action For Health in Diabetes (Look AHEAD) study has shown that weight loss via diet and exercise improves glycaemic control.(21) However, our study found that unlike medical therapy, the improvements in glycaemic control and weight achieved by MBS are sustained over a one-year period. Two recent randomised controlled trials comparing bariatric surgery with intensive medical therapy in T2DM likewise reported that bariatric surgery was more effective in achieving good glycaemic control than intensive medical therapy alone. ${ }^{(22,23)}$

As the present study was a prospective observational study in a clinical care setting, and not a randomised controlled trial, there were more patients in the GB group $(n=14)$ than the SG group $(n=5)$. Since current evidence suggests that bypass procedures offer greater DM benefits, ${ }^{(20,24)}$ patients with poorly controlled T2DM were advised to undergo GB. This explains the higher $\mathrm{HbA1c}$ baseline in the GB group $(8.5 \%)$ as compared to the SG group $(7.3 \%)$. Our study suggests that although the patients in the GB group had worse glycaemic control at baseline,
Table III. Medication use at baseline and 12 months.

\begin{tabular}{lcc}
\hline Medication & \multicolumn{2}{c}{ No. (\%) } \\
\cline { 2 - 3 } & Baseline & At 12 mths \\
\hline Antidiabetic & $16(84.2)$ & $5(26.3)$ \\
Metformin & $13(68.4)$ & $2(10.5)$ \\
Sulphonylurea & $7(36.8)$ & $1(5.3)$ \\
DPP4 inhibitors & $9(47.4)$ & 0 \\
Insulin & & \\
No. of DM medications & $2(10.5)$ & $14(73.7)$ \\
0 & $3(15.8)$ & $3(15.8)$ \\
1 & $6(31.6)$ & $1(5.3)$ \\
2 & $7(36.8)$ & 0 \\
3 & $1(5.3)$ & \\
4 & & $6(31.6)$ \\
Antihypertensive & $14(73.7)$ & $4(21.1)$ \\
ACEi/ARB & $7(36.8)$ & $2(10.5)$ \\
Calcium channel blocker & $6(31.6)$ & \\
Beta-blocker & & $4(21.1)$ \\
Lipid-lowering & $13(68.4)$ & 0 \\
Statins & $1(5.3)$ & \\
Fibrate & &
\end{tabular}

ACEi: angiotensin-converting enzyme inhibitor; ARB: angiotensin receptor blocker; DM: diabetes mellitus; DPP4: dipeptidyl peptidase-4

they still managed to achieve a similar degree of good glycaemic control as the SG group, despite both groups achieving a similar degree of weight loss. The rise in HDL cholesterol and reduction in triglycerides levels were also more significant in the GB group. While this difference could be attributed to the unbalanced sample size of the two groups and the higher HbA1c baseline in the GB group, there is growing evidence that suggests GB may offer greater metabolic benefits than SG.

In a recent study on a Taiwanese population, 60 patients with T2DM were randomised to undergo either GB or SG. ${ }^{(24)}$ At one year, there was a statistically greater number of patients in the GB group who achieved DM remission as compared to the SG group (93\% vs. $47 \%$ ). Greater weight loss and reduction in waist circumference and blood lipid levels were also seen in the GB group. ${ }^{(24)}$ This phenomenon has been well described in other studies, which indicate that gastrointestinal bypass techniques improve glucose homeostasis through mechanisms beyond weight loss and reduced calorie intake. ${ }^{(25)}$

In Singapore, the difference in the prevalence of T2DM in each ethnicity is paralleled by the degree of abdominal fatness (measured by waist-hip ratio), with Indians having the highest prevalence for both. ${ }^{(13)}$ According to the 2010 National Health Survey, ${ }^{(13)}$ nearly one-third (32\%) of patients with DM have poor glycaemic control $(\mathrm{HbA} 1 \mathrm{c}>8.0 \%)$, of which almost half (47.6\%) were Malays, and only $24.9 \%$ were Chinese. In our study, $47 \%$ of our patients were of Chinese ethnicity, while $26 \%$ were Malays and $21 \%$ Indians. The proportionate discrepancy in poor glycaemic control and the number of patients in each ethnicity who underwent MBS as a treatment for T2DM could indicate that a gap exists in the care of obese patients 
with T2DM, which could be addressed by raising awareness of formal weight loss intervention (including MBS) as a treatment option in the management of T2DM.

Apart from the inherent limitation of our study due to its observational nature, other limitations include a small sample size. The present study may not be adequately powered to detect significant differences in other metabolic parameters, especially between GB and SG, as well as among the various ethnic groups. Despite these limitations, we observed a significant reduction in $\mathrm{HbA1c}$ and triglycerides levels, and an increase in HDL levels in the GB group when compared to the SG group. We also believe that the results of our study corroborate the fact that the international recommendation of MBS as an effective treatment modality for obese patients with T2DM is relevant to our local population. Consistent with other studies, our study suggests that MBS is effective in not only achieving significant weight loss, but also improving the glycaemic control of patients with T2DM. This improvement is associated with a reduction in medication use and other cardiovascular risk factors, particularly a significant increment of HDL cholesterol levels, which may reduce cardiovascular disease burden in the future. A randomised controlled trial comparing GB and SG, using a larger sample population powered to look into the effects of both procedures on glycaemic control in each ethnic group, will be useful.

\section{REFERENCES}

1. Chan JM, Rimm EB, Colditz GA, Stampfer MJ, Willett WC. Obesity, fat distribution, and weight gain as risk factors for clinical diabetes in men. Diabetes Care 1994; 17:961-9.

2. Ma S, Cutter J, Tan CE, Chew SK, Tai ES. Associations of diabetes mellitus and ethnicity with mortality in a multiethnic Asian population: data from the 1992 Singapore National Health Survey. Am J Epidemiol 2003; 158:543-52.

3. Stratton IM, Adler AI, Neil HA, et al. Association of glycaemia with macrovascular and microvascular complications of type 2 diabetes (UKPDS 35): prospective observational study. BMJ 2000; 321:405-12.

4. Laiteerapong N, John PM, Nathan AG, Huang ES. Public health implications of recommendations to individualize glycaemic targets in adults with diabetes. Diabetes Care 2013; 36:84-9.
5. Pories WJ, Swanson MS, MacDonald KG, et al. Who would have thought it? An operation proves to be the most effective therapy for adultonset diabetes mellitus. Ann Surg 1995; 222:339-50.

6. Sjöström L, Lindroos AK, Peltonen $M$, et al. Lifestyle, diabetes, and cardiovascular risk factors 10 years after bariatric surgery. $\mathrm{N}$ Engl J Med 2004; 351:2683-93.

7. Sjöström L, Narbro K, Sjöström CD, et al. Effects of bariatric surgery on mortality in Swedish obese subjects. N Engl J Med 2007; 357:741-52.

8. Sjöström L, Peltonen M, Jacobson P, et al. Bariatric surgery and long-term cardiovascular events. JAMA 2012; 307:56-65.

9. Dixon JB, Zimmet $\mathrm{P}$, Alberti KG, Rubino F, International Diabetes Federation Taskforce on Epidemiology and Prevention. Bariatric surgery: an IDF statement for obese Type 2 diabetes. Diabet Med 2011; 28:628-42.

10. Lomanto D, Lee WJ, Goel R, et al. Bariatric surgery in Asia in the last 5 years (2005-2009). Obes Surg 2012; 22:502-6.

11. Lee WJ, Chong K, Lee YC, et al. Effects of obesity surgery on type 2 diabetes mellitus Asian patients. World J Surg 2009; 33:1895-903.

12. National Health Survey. 2004 Epidemiology and Disease Control Department Ministry of Health, Singapore.

13. National Health Survey. 2010 Epidemiology and Disease Control Department Ministry of Health, Singapore.

14. Kiong KL, Ganesh R, Cheng AK, Lekshiminarayanan R, Lim SC. Early improvement in type 2 diabetes mellitus post Roux-en-Y gastric bypass in Asian patients. Singapore Med J 2010; 51:937-43.

15. Ministry of Health. Clinical Practice Guidelines 5/2004 Obesity. Singapore: Ministry of Health.

16. Lee SY, Lim CH, Pasupathy S, et al. Laparoscopic sleeve gastrectomy: a novel procedure for weight loss. Singapore Med J 2011; 52:794-800.

17. DeMaria EJ. Bariatric surgery for morbid obesity. N Engl J Med 2007; 356:2176-83.

18. Buse JB, Caprio S, Cefalu WT, et al. How do we define cure of diabetes? Diabetes Care 2009; 32:2133-5.

19. Action to Control Cardiovascular Risk in Diabetes Study G, Gerstein $\mathrm{HC}$, Miller ME, et al. Effects of intensive glucose lowering in type 2 diabetes. N Engl J Med 2008; 358:2545-59.

20. Buchwald H, Estok R, Fahrbach K, et al. Weight and type 2 diabetes after bariatric surgery: systematic review and meta-analysis. Am J Med 2009; 122:248-56.e5.

21. Look AHEAD Research Group, Wadden TA, West DS, et al. The Look AHEAD study: a description of the lifestyle intervention and the evidence supporting it. Obesity (Silver Spring) 2006; 14:737-52.

22. Mingrone G, Panunzi S, De Gaetano A, et al. Bariatric surgery versus conventional medical therapy for type 2 diabetes. N Engl J Med 2012; 366:1577-85.

23. Schauer PR, Kashyap SR, Wolski K, et al. Bariatric surgery versus intensive medical therapy in obese patients with diabetes. N Engl J Med 2012; 366:1567-76.

24. Lee WJ, Chong K, Ser KH, et al. Gastric bypass vs sleeve gastrectomy for type 2 diabetes mellitus: a randomized controlled trial. Arch Surg 2011; 146:143-8.

25. Rubino F, R'bibo S L, del Genio F, Mazumdar M, McGraw TE. Metabolic surgery: the role of the gastrointestinal tract in diabetes mellitus. Nat Rev Endocrinol 2010; 6:102-9. 